\title{
THE IMPACT OF FEEDBACK AND WARNING ON RETRIEVAL-ENHANCED SUGGESTIBILITY
}

\author{
Xilei Wang, Xueying Li, Wenwu Dai, \& Ning Jia \\ Hebei Normal University (China)
}

\begin{abstract}
Retrieval practice can exacerbate eyewitness' susceptibility to subsequent misinformation and then produces more false memories is known as Retrieval-Enhanced Suggestibility (RES). In the field of judicial psychology, eyewitness testimony plays a crucial role, and even directly affects the judgment of the suspect. The eyewitnesses may be interfered with by other irrelevant information or repeated inquiries by the police, thus causing misinformation interference from the original information. In all three experiments, this study uses pictures of Chinese criminal investigation dramas as experimental materials. This study examines the mechanism of RES effect by manipulating the feedback from retrieval and warning. The results show that: (1) There is still a significant RES effect on the Chinese context; (2) Both feedback and warning play an important role in the generation of RES. Among them, the feedback enhanced the participant' memory of the original information and reduced the credibility of misinformation. Thus, the RES effect is reduced; (3) Warnings reduce the credibility of all narrative information, thereby reducing the RES effect. In short, both feedback and warning can reduce the RES effect, but the effect of feedback is more positive and precise.
\end{abstract}

Keywords: Retrieval-enhanced suggestibility, feedback, warning.

\section{Introduction}

Witnesses are sometimes more susceptible to misinformation if their memory for the witnessed event had been tested prior to misinformation exposure. Retrieval practice can exacerbate eyewitness' susceptibility to subsequent misinformation and then produces more false memories is known as Retrieval-Enhanced Suggestibility (RES effect) (Chan, Thomas, \& Bulevich, 2009). Factors that affect discrepancy detection include memory strength for the original event, the elapsed time between viewing the original event and the memory test, the subtlety of the misinformation, and warnings about misinformation (Leding \& Antonio, 2019). So, study the impact of feedback and warning on the RES effect. Summarizing the previous literature, it is found that warning can effectively reduce the RES effect (Anna, Melanie \& Harald, 2018; Thomas, Bulevich \& Chan, 2010; Manley \& Chan, 2019). Feedback is a kind of information that allows learners to determine, add, rewrite, adjust, or reconstruct the information about their memory (Hattie \& Timperley, 2007). Many studies have found that feedback has an impact on misleading information and feedback can improve the learning effect of learners (Merckelbach, Dalsklev, Daniël, Boskovic, \& Otgaar, 2018; Nicklin \& Williams, 2011).

\section{Experiment 1}

This research used pictures of Chinese criminal investigation dramas as experimental materials. Based on the classic RES paradigm, the overall credibility of the narrative information is judged. The credibility of the narrative information is judged after the end of the final memory test stage, and the subjects are required to judge the credibility of the narrative information. This experiment had 2 (Group: tested participants vs. control participants) $\times 3$ (Item type: consistent items, neutral items, misled items) mixed design. There were 22 participants in the tested condition and 22 in the control condition. For misinformation recall, an independent samples $t$-test showed that the between-subjects RES effect was significant, $t=-3.52, p=0.001, d=1.068$. For correct recall, a 2 (Group: tested participants vs. control participants) $\times 3$ (Item type: consistent items, neutral items, misled items) ANOVA yielded significant main effects for Item Type, $F(2,41)=17.068, p<0.001, \eta_{p}{ }^{2}=0.454$, and the interaction between these variables, $F(2,41)=8.126, p=0.001, \eta_{p}{ }^{2}=0.284$. The interaction showed that tested participants were more likely to recall correct for consistent items than control participants, $F(1,42)=8.687, p=0.005, \eta_{p}{ }^{2}=0.171$, 
there was a testing effect; control participants were more likely to recall correct for misled items than tested participants, $F(1,42)=7.827, p=0.008, \eta_{p}{ }^{2}=0.157$. Tested participants were more recalling the correct event details for neutral items than for misled items, $p<0.001$. Tested participants were more recalling the correct event details for consistent items than for misled items, $p<0.001$. Tested participants were more recalling the correct event details for consistent items than for neutral items, $p=0.001$. Control participants were not significant. For credibility ratings, an independent samples $t$-test showed that there is no significant difference in credibility ratings between the tested participants and the control participants. This may be due to the participants' insufficient learning of the picture information, so that the participants did not realize the inconsistency between the narrative information and the picture information.

\section{Experiment 2}

This experiment studies the overall credibility of misleading information and narrative information by manipulating feedback. Experiment had 2 (Group: feedback participants vs. control participants) $\times 3$ (Item type: consistent items, neutral items, misled items) mixed design. There were 22 participants in the feedback condition and 22 in the control condition. The procedure was basically the same as Experiment 1, except that the feedback stage was added. Positive and negative feedback was given after the retrieval practice. Give feedback question by question. For misinformation recall, an independent samples $t$-test showed that the feedback participants were significantly lower than control participants, $p<0.001, d=1.427$, this indicates that feedback can improve the learning level of the subjects on the original information, and thus can better reduce the influence of misleading information on themselves. For correct recall, a 2 (Group: feedback participants vs. control participants) $\times 3$ (Item: consistent items, neutral items, misled items) ANOVA yielded significant main effects for Group, $F(1,42)=7.929, p=0.007, \eta_{p}{ }^{2}=0.15$. Feedback participants out-performed the control participants. For credibility ratings, an independent samples $t$-test showed that feedback participants more less than control participants, $p=0.012, d=0.790$. That this research not only found that feedback can reduce RES effect, but also found that feedback can improve the participant's learning level of the original information, so that the participant can find the difference between the original information and the narrative information, thereby reducing the credibility of the narrative information.

\section{Experiment 3}

The experiment explored the impact of changing the overall credibility of the participants' narrative information through warnings on recalling misleading information. Experiment had 2 (Group: warning participants vs. control participants) $\times 3$ (Item type: consistent items, neutral items, misled items) mixed design. There were 22 participants in the warning condition and 22 in the control condition. The experiment procedure was basically the same as Experiment 1, except that the warning stage was added. Before the narrative information stage, the subjects were randomly assigned to the warning group or the control group. For misinformation recall, an independent samples $t$-test showed that the warning participants were significantly lower than control participants, $p<0.001, d=1.169$. For correct recall, a 2 (Group: tested participants vs. control participants) $\times 3$ (Item type: consistent items, neutral items, misled items) ANOVA yielded significant main effects for Item Type, $F(2,41)=30.116, p<0.001, \eta_{p}{ }^{2}=0.595$, and the interaction between these variables, $F(2,41)=10.546, p<0.001, \eta_{p}{ }^{2}=0.340$. The interaction showed that warning participants were more likely to recall correct for misled items than control participants, $F(1,42)=14.779, p<0.001, \eta_{p}{ }^{2}=0.260$, control participants were more likely to recall correct for consistent items than warning participants, $F(1,42)=6.505, p=0.014, \eta_{p}{ }^{2}=0.134$, this shows that because the credibility of the narrative information is manipulated through warnings, the subjects can reject the misleading information and respond according to the original information they remember. Warning participants were more recalling the correct event details for consistent items than for misled items, $\mathrm{p}=0.020$. there were no significant differences between the other items. control participants were more recalling the correct event details for neutral, $p<0.001$, and consistent, $p<0.001$, items than for misled items. control participants were more recalling the correct event details for consistent items than for misled items, $p=0.001$. For credibility ratings, an independent samples $t$-test showed that warning participants more less than control participants, $p=0.003, d=0.939$. Warnings can reduce the credibility of the narrative information, this shows that the warning will make subjects suspicious whether the information is correct or misleading. 


\section{Conclusions}

First, the RES effect is stable in Chinese materials. Then, both feedback and warning can reduce the recall of misleading information and the RES effect. However, their processing mechanism is different. Feedback reduces the credibility of the narrative information by discovering the difference between the original information and the narrative information, thereby reducing the RES effect. However, warning reduces the learning of narrative information by completely questioning the narrative information, thereby reducing the RES effect.

\section{References}

Anna, S., Melanie, S., \& Harald, M. (2018). Warnings to counter choice blindness for identification decisions: warnings offer an advantage in time but not in rate of detection. Frontiers in Psychology, 9, 981.

Chan, J. C. K., Thomas, A. K., \& Bulevich, J. B. (2009). Recalling a witnessed event increases eyewitness suggestibility: The reversed testing effect. Psychological Science, 20(1), 66-73.

Hattie, J., \& Timperley, H. (2007). The Power of Feedback. Review of Educational Research, 77(1), 81-112.

Leding, J. K., \& Antonio, L. (2019). Need for cognition and discrepancy detection in the misinformation effect. Journal of Cognitive Psychology, 31(4), 409-415.

Manley, K. D., \& Chan, J. C. K. (2019). Does retrieval enhance suggestibility because it increases perceived credibility of the postevent information?. Journal of Applied Research in Memory and Cognition, 8(3), 355-366.

Merckelbach, H., Dalsklev, M., Daniël van Helvoort, Boskovic, I., \& Otgaar, H. (2018). Symptom selfreports are susceptible to misinformation. Psychology of Conousness: Theory, Research, and Practice, 36(3), 604-616.

Nicklin, J. M., \& Williams, K. J. (2011). Self-regulation of goals and performance: effects of discrepancy feedback, regulatory focus, and self-efficacy. Psychology, 2(3), 187-201.

Thomas, A. K., Bulevich, J. B., \& Chan, J. C. K. (2010). Testing promotes eyewitness accuracy with a warning: implications for retrieval enhanced suggestibility. Journal of Memory and Language, 63(2), 149-157. 